\title{
Suborbital graphs of a power subgroup of the modular group
}

\author{
Zeynep Şanlı ${ }^{1}$, Tuncay Köroğlu ${ }^{2}$ and Bahadır Ö̈gür Güler ${ }^{2}$ \\ ${ }^{1}$ Karadeniz Technical University, Institute of Natural Sciences, Trabzon, Turkey \\ ${ }^{2}$ Karadeniz Technical University, Department of Mathematics, Trabzon, Turkey \\ Received: 3 August 2017, Accepted: 26 August 2018 \\ Published online: 21 February 2018.
}

\begin{abstract}
In this paper, we define an invariant equivalence relation by using the group $\Gamma(2)$. Then we investigate some combinatorial properties of subgraphs of $\Gamma^{2}$.
\end{abstract}

Keywords: Modular group, imprimitive action, suborbital graphs.

\section{Introduction}

\subsection{Motivation}

Using the notion of the imprimitive action for an invariant equivalence relation on $\widehat{\mathbb{Q}}$ by the congruence subgroup $\Gamma_{0}(n)$, Jones, Singerman and Wicks obtained suborbital graphs of the modular group $\Gamma$ and showed that these graphs are the generalization of the well-known Farey graph[8]. Then Akbas found certain relationship between the lenghts of circuits in these graphs and periods of elliptic elements of the group $\Gamma_{0}(n)[1]$. This is important taking into account that the elliptic elements are one of the invariants of the group. Hence, suborbital graphs can be viewed as a tool to investigate permutation groups in terms of combinatorics[5].

Actually, the suborbital graphs of the group $\Gamma^{2}$ were studied in[7] for the relation $\Gamma_{\infty}^{2} \lesseqgtr \Gamma_{0}^{2}(n) \lesseqgtr \Gamma^{2}$ with $n \in \mathbb{N}$. In here, taking $\Gamma(2)$ instead of $\Gamma_{0}^{2}(n)$, we investigate some combinatorial properties of the newly constructed subgraphs of $\Gamma(2)$ different from[7]. We can summarize the cause of this choice as follows.

Congruence subgroups of $\Gamma$ are very important in number theory; they all have finite index in $\Gamma$, but not every subgroup of finite index is a congruence subgroup. Some of them have a special interest. $\operatorname{In}[16]$, Singerman showed that $\Gamma_{0}(2)$ is isomorphic to the universal tessellation $\Gamma(2, \infty, \infty)$. He pointed that this is a chance taking into account the difficulties of construction of universal $n$-gonal tessellations. It is known that the groups $\Gamma(2, \infty, n)$ are Hecke groups and more complicated than the modular group $\Gamma(2,3, \infty)$.

Furthermore, the plane trees, the maps of genus 0 with a single face, can be probably seen as the simplest class of bipartite maps. In[9], $\Gamma(2)$ is given the automorphism group of the universal bipartite map $\hat{\mathfrak{B}}$ on $\mathbb{H}$. It is used as an illustration to emphasized the connections between maps on surfaces, permutations, Riemann surfaces. 
From this point of view, to collect new results about on $\Gamma(2)$, we used the relation $\Gamma_{\infty}^{2} \lesseqgtr \Gamma(2) \lesseqgtr \Gamma^{2}$ for the imprimitive action in this paper.

\subsection{Preliminaries}

Define $\Gamma^{m}$ as the subgroup of $\Gamma$ generated by the $m^{\text {th }}$ powers of all elements of $\Gamma$. Especially, $\Gamma^{2}$ and $\Gamma^{3}$ have been studied extensively by [11][12][13]. It turns out that,

$$
\Gamma^{2}=\left\{\left(\begin{array}{ll}
a & b \\
c & d
\end{array}\right) \in \Gamma: a b+b c+c d \equiv 0(\bmod 2)\right\}
$$

by Rankin [14]. From the equation $a b+b c+c d \equiv 0(\bmod 2)$, we see that at least one of the letters $a, b, c, d$ must be even. Suppose first that $a=2 a_{0}$. Then using the determinant, we have that $b$ and $c$ are odd. So, $d$ must be odd as well. Hence, we get the element of $\Gamma^{2}$ as the matrices $\left(\begin{array}{cc}2 a & b \\ c & d\end{array}\right)$. Similarly, supposing $d=2 d_{0}$, we can get the elements of the form $\left(\begin{array}{ll}a & b \\ c & 2 d\end{array}\right)$. Lastly, if $a$ or $d$ is not even, then both $b$ and $c$ will be even. To sum up, $\Gamma^{2}$ has three types of elements

$$
\left(\begin{array}{cc}
2 a & b \\
c & d
\end{array}\right),\left(\begin{array}{cc}
a & 2 b \\
2 c & d
\end{array}\right),\left(\begin{array}{ll}
a & b \\
c & 2 d
\end{array}\right)
$$

where $b, c$ and $d$ of the first, $a$ and $d$ of the second and $a, b, c$ of the third matrix are odd.

In this study, we also use congruance subgroup $\Gamma(2)$ of the modular group, so we give some information about this group. For any positive integer $n$, the group showed $\Gamma(n)$ is defined as follow:

$$
\Gamma(n)=\left\{\left(\begin{array}{ll}
a & b \\
c & d
\end{array}\right) \in \Gamma: a \equiv d \equiv 1(\bmod n), b \equiv c \equiv 0(\bmod n)\right\} .
$$

For $n=2$, the group $\Gamma(2)$ is generated by three elements

$$
\left(\begin{array}{ll}
1 & 2 \\
0 & 1
\end{array}\right),\left(\begin{array}{ll}
3 & -2 \\
2 & -1
\end{array}\right),\left(\begin{array}{cc}
-1 & 0 \\
0 & -1
\end{array}\right)
$$

and its cusps are $0,1, \infty$.

\section{The Action of $\Gamma^{2}$ on $\widehat{\mathbb{Q}}$}

Every element of $\hat{\mathbb{Q}}$ can be represented as a reduced fraction $\frac{x}{y}$, with $x, y \in \mathbb{Z}$ and $(x, y)=1$; since $\frac{x}{y}=\frac{-x}{-y}$, this representation is not unique. We represent $\infty$ as $\frac{1}{0}=\frac{-1}{0}$. The action $z \rightarrow \frac{a z+b}{c z+d}$ of $\Gamma^{2}$ on $\hat{\mathbb{Q}}$ now becomes

$$
\left(\begin{array}{ll}
a & b \\
c & d
\end{array}\right): \frac{x}{y} \rightarrow \frac{a x+b y}{c x+d y}
$$

Lemma 1.[7]

(i) The action of $\Gamma^{2}$ on $\hat{\mathbb{Q}}$ is transitive. 
(ii) The stabilizer of a point is in infinite cyclic group.

Proposition 1.[4] Let $(G, \Omega)$ be transitive. Then $(G, \Omega)$ is primitive if and only if $G_{\alpha}$, the stabilizer of a point $\alpha \in \Omega$, is a maximal subgroup of $G$ for each $\alpha \in \Omega$.

Indeed, suppose that $G_{\alpha}<H<G$. Since $G$ acts transitively, every element of $\Omega$ has the form $g(\alpha)$ for some $g \in G$. One easily checks that there is a well-defined $G$ - invariant equivalence relation $\approx$ on $\Omega$, given by $g(\alpha) \approx g^{\prime}(\alpha)$ if and only if $g^{\prime} \in g H$.

We now apply these ideas to the case where $G$ is $\Gamma^{2}$, and $\Omega$ is $\widehat{\mathbb{Q}}$. Here $\Gamma_{\infty}^{2}$, the stabilizer of $\infty$, is the subgroup of $\Gamma^{2}$ generated by $\left(\begin{array}{ll}1 & 2 \\ 0 & 1\end{array}\right)$, so by finding subgroups $H$ of $\Gamma^{2}$ containing $\Gamma_{\infty}^{2}\left(\right.$ or equivalently, containing $\left.\left(\begin{array}{ll}1 & 2 \\ 0 & 1\end{array}\right)\right)$, we can produce $\Gamma^{2}$ - invariant equivalence relations on $\hat{\mathbb{Q}}$. From the matrix $\left(\begin{array}{ll}1 & 2 \\ 0 & 1\end{array}\right)$, some obvious choices for $H$ are the congruence subgroups

$$
\Gamma(2)=\{A \in \Gamma: A \equiv I(\bmod 2)\} .
$$

Clearly, $\Gamma_{\infty}^{2}<\Gamma(2)<\Gamma^{2}$, so $\Gamma^{2}$ acts imprimitively on $\widehat{\mathbb{Q}}$. Let $\approx$ denote $\Gamma^{2}-$ invariant equivalence relation induced on $\widehat{\mathbb{Q}}$ by $\Gamma(2)$. If $v=\frac{r}{s}$ and $w=\frac{x}{y}$ are elements of $\hat{\mathbb{Q}}$, then $v=g(\infty)$ and $w=g^{\prime}(\infty)$ for elements $g, g^{\prime} \in \Gamma^{2}$ of the form

$$
g=\left(\begin{array}{ll}
r & k \\
s & l
\end{array}\right),\left(\begin{array}{ll}
x & z \\
y & t
\end{array}\right)
$$

now $v \approx w$ if and only if $g^{-1} g^{\prime} \in H=\Gamma(2)$, and since $g^{-1}=\left(\begin{array}{cc}l & -k \\ -s & r\end{array}\right)$ we see that $v \approx w$ if and only if

$$
\begin{aligned}
& l x-k y \equiv r t-s z \equiv \pm 1(\bmod 2) \\
& l z-k t \equiv r y-s x \equiv \pm 1(\bmod 2)
\end{aligned}
$$

To put this another way, $v=\frac{r}{s}$ and $w=\frac{x}{y}$ are equivalent if and only if they "have the same reduction mod2", that is,

$$
x \equiv u r \text { and } y \equiv u s(\bmod 2)
$$

for some unit $u \in U_{2}$.

By our general discussion of imprimitivity, the number $\Psi(2)$ of equivalence classes under $\approx$ is given by

$$
\Psi(2)=\left|\Gamma^{2}: \Gamma(2)\right|=3
$$

\section{Suborbital Graphs for $\Gamma^{2}$ on $\hat{\mathbb{Q}}$}

Let $(G, \Omega)$ be a transitive permutation group. Then $G$ acts on $\Omega \times \Omega$ by

$$
g:(\alpha, \beta) \rightarrow(g(\alpha), g(\beta))
$$

$(g \in G, \alpha, \beta \in \Omega)$. The orbits of this action are called suborbitals of $G$, that containing $(\alpha, \beta)$ being denoted by $O(\alpha, \beta)$ From $O(\alpha, \beta)$ we can form a suborbital graph $G(\alpha, \beta)$ : Its vertices are the elements of $\Omega$, and there is a directed edge from $\gamma$ to the $\delta$ if $(\gamma, \delta) \in O(\alpha, \beta)$. 
Clearly $O(\alpha, \beta)$ is also a suborbital graph, and it is either equal to our disjoint from $O(\alpha, \beta)$. In the latter case, $G(\alpha, \beta)$ is just $G(\alpha, \beta)$ with the arrows reversed, and we call $G(\alpha, \beta)$ and $G(\beta, \alpha)$ paired suborbital graphs. In the former case, $G(\alpha, \beta)=G(\beta, \alpha)$ and the graphs consists of pairs of oppositely directed edges; it is convenient to replace each such pair by a single undirected edge, so that we have an undirected graph which we call self-paired. These ideas were first introduced by Sims[15] and are also described in a paper by Newmann[10] and in books Tsuzuku[17], Biggs and White[4], the emphasis being on applications to finite groups. The reader is also refereed to [2][3][6][7] for some relevant previous work on suborbital graphs.

Theorem 1. $\frac{r}{s} \rightarrow \frac{x}{y} \in G_{u, 2}$ if and only if

$$
x \equiv \pm u r(\bmod 4), x \equiv \pm u s(\bmod 2) \text { and } r y-s x= \pm 2
$$

Proof. By the transitivity of $\Gamma^{2}$, without loos of generality, we assume that $\frac{r}{s}<\frac{x}{y}$ where all letters are positive integers. Thus, we have that $r y-s x<0$. Since $\frac{r}{s} \rightarrow \frac{x}{y} \in G_{u, 2}$, there exist some $T=\left(\begin{array}{ll}a & b \\ c & d\end{array}\right) \in \Gamma^{2}$ such that $T\left(\frac{1}{0}, \frac{u}{2}\right)=\left(\frac{r}{s}, \frac{x}{y}\right)$. As $r y-s x<0$, the multiplication of $\left(\begin{array}{ll}a & b \\ c & d\end{array}\right)\left(\begin{array}{ll}1 & u \\ 0 & 2\end{array}\right)$ is equal to $\left(\begin{array}{ll}-r & x \\ -s & y\end{array}\right)$ or $\left(\begin{array}{l}r-x \\ s-y\end{array}\right)$. If the first case is valid, we have that $a=-r, c=-s, a u+2 b=x, c u+2 d=y$ and $r y-s x=-2$. That is, $x \equiv-u r(\bmod 2)$ and $y \equiv-u s(\bmod 2)$. Since $s$ is even we see that $b$ and $c$ must be even because $T\left(\frac{1}{0}\right)=\frac{-r}{-s}=\frac{a}{c}$. Since $b$ is even, we have that $x \equiv-u r(\bmod 4)$ and $y \equiv-u s(\bmod 2)$.

In the opposite direction, we shall prove the theorem for minus sign. Suppose that $x \equiv-u r(\bmod 4), y \equiv-u s(\bmod 2)$ and $r y-s x=-2$. In this, there exist integers $b, d$ such that $x=-u r-4 b, y=-u s-2 d$. So, it is clear that $\left(\begin{array}{c}-r-2 b \\ -s-d\end{array}\right) \in \Gamma^{2}$ which means $\frac{r}{s} \rightarrow \frac{x}{y} \in G_{u, 2}$. Because $-2=r y-s x=r(-u s-2 d)-s(-u r-4 b)$. This implies $r d-2 b s=1$. We can illustrate one example for this subgraph obtained from elements $A=\left(\begin{array}{l}3-2 \\ 2-1\end{array}\right)$ and $B=\left(\begin{array}{l}5-8 \\ 2-3\end{array}\right)$ with figure1.

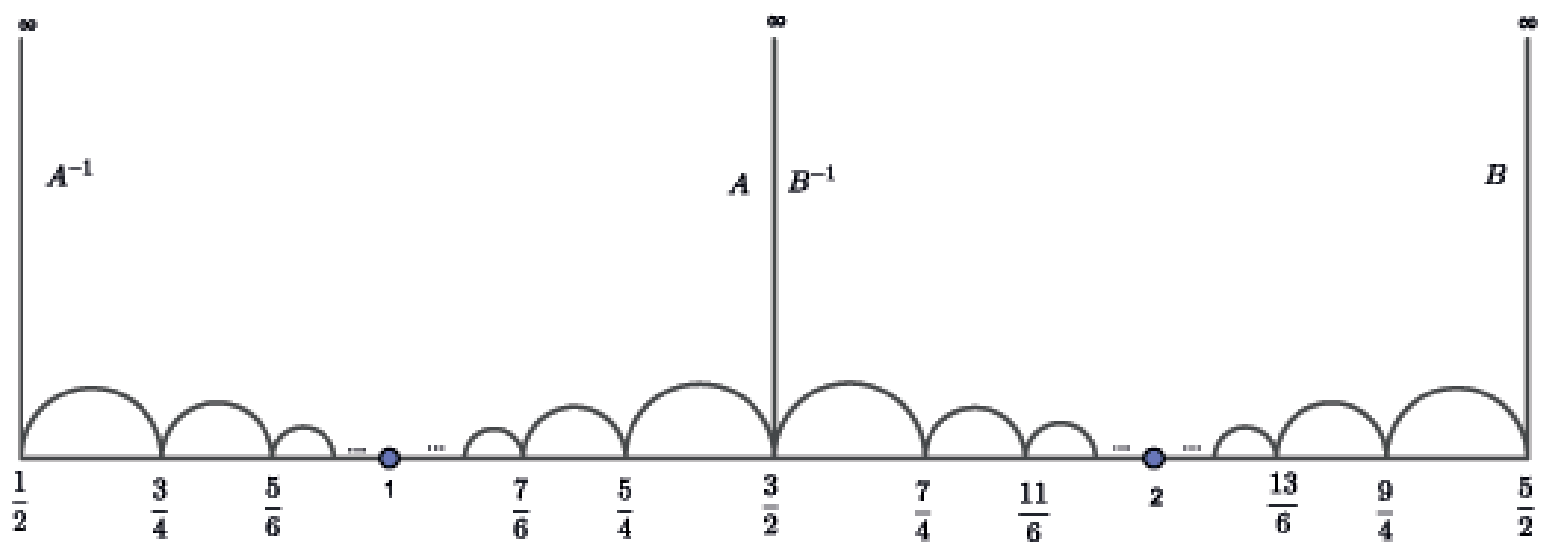

Fig. 1: The paths of $\Gamma^{2}$.

Theorem 2. Let $T=\left(\begin{array}{ll}a & b \\ c & d\end{array}\right) \in \Gamma^{2}$. Then $\frac{1}{2} \rightarrow \frac{1}{4} \in F^{2}$ if and only if $T=\left(\begin{array}{cc}4 n+1 & -2 n \\ 16 n+2 & 1-8 n\end{array}\right)$ with $n \in \mathbb{Z}$. 
Proof. Let $T\left(\frac{1}{2}\right)=\frac{1}{4}=\frac{a+2 b}{c+2 d}$. Because $c+2 d=a+2 b+2, T$ is $\left(\begin{array}{rr}2 a-1 & 1-a \\ 2 c & 2-c\end{array}\right)$. Since $T \in \Gamma, 4 a-c=3$ and so $a=4 k+1$, $k \in \mathbb{Z}$. Therefore, $T=\left(\begin{array}{cc}2 k+1 & -k \\ 8 k+2 & 1-4 k\end{array}\right)$. On the other hand, because $T \in \Gamma^{2}(2 k+1)(-k)+(-k)(8 k+2)+(8 k+$ $2)(1-4 k) \equiv 0(\bmod 2)$.From the last congruence, $k \equiv 0(\bmod 2)$. So, $k=2, n \in \mathbb{Z}$. As a result, $T$ is in the form $\left(\begin{array}{cc}4 n+1 & -2 n \\ 16 n+2 & 1-8 n\end{array}\right)$. The opposite is obvious.

Theorem 3. Let $n \geq 2, n \in \mathbb{Z}$. The transformations of the matrices $T=\left(\begin{array}{cc}4 n+1 & -2 n \\ 16 n+2 & 1-8 n\end{array}\right)$ are hyperbolic transformations and fixed points of these transformation are attracting fixed points.

Proof. The trace of the transformations of the matrices $T=\left(\begin{array}{cc}4 n+1 & -2 n \\ 16 n+2 & 1-8 n\end{array}\right)$ is equal to $|2-4 n|$. So, when $n=0$ and 1 , these transformations are parabolic, when $n \geq 2$, they are hyperbolic, because of $|2-4 n| \geq 2$. The fixed points of these transformations,

$$
z_{1,2}=\frac{3 n \pm \sqrt{n^{2}-n}}{8 n+1}
$$

Therefore, $T^{\prime}(z)=\frac{1}{[(16 n+2 z+1-8 n)]^{2}}$ and so $T^{\prime}\left(z_{1,2}\right)=\left(\frac{1}{1-2 n \pm \sqrt{n^{2}-n}}\right)<1$, with $\forall n \geq 2$. Thus, these fixed points are attracting fixed points.

Theorem 4. Let $T_{n}:=\left(\begin{array}{cc}a n+1 & b n \\ c n & d n+1\end{array}\right) \in \Gamma(n), c \neq 0$ and $n \geq 2$. Then $\left|T_{n}(\infty)-T_{n}^{2}(\infty)\right| \leq \frac{1}{2 n}$.

Proof. For $T=\left(\begin{array}{cc}a n+1 & b n \\ c n & d n+1\end{array}\right), T^{2}$ is equal to $\left(\begin{array}{cc}(a n+1)^{2}+b c n^{2} & b n(a n+d n+2) \\ c n(a n+d n+2) & b c n^{2}+(d n+1)^{2}\end{array}\right)$. Therefore,

$$
T(\infty)=\frac{a n+1}{c n} \text { and } T^{2}(\infty)=\frac{(a n+1)^{2}+b c n^{2}}{c n(a n+d n+2)} .
$$

So,

$$
\left|T(\infty)-T^{2}(\infty)\right|=\left|\frac{a n+1}{c n}-\frac{(a n+1)^{2}+b c n^{2}}{c n(a n+d n+2)}\right|=\left|\frac{1}{c n^{2}(a+d)+2 c n}\right|=\frac{1}{|c| n} \frac{1}{|n(a+d)+2|} .
$$

Let $M$ be $\frac{1}{|n(a+d+2)|}$. In this case, when $n \geq 2,|n(a+d)+2| \geq|2(a+d)+2|=2|a+d+1| \geq 2$. So, $M$ becomes less than $\frac{1}{2}$, when $n \geq 2$ and $c \neq 0$. As a result $\left|T(\infty)-T^{2}(\infty)\right| \leq \frac{1}{2 n}$. .

From this theorem, we can easily say that, the maximum value of distance of two vertices $T(\infty)$ and $T^{2}(\infty)$ is $\frac{1}{4}$ for all $T \in \Gamma(2)$. The following is a result of this theorem.

Corollary 1. Let $T=\left(\begin{array}{cc}4 n+1 & -2 n \\ 16 n+2 & 1-8 n\end{array}\right)$. Then $\left|T(\infty)-T^{2}(\infty)\right| \leq \frac{1}{4}$.

Proof. Because $T(\infty)=\frac{4 n+1}{16 n+2}$ and $T^{2}(\infty)=\frac{-16 n^{2}+4 n+1}{-64 n^{2}+24 n+4}$, then

$$
\left|T(\infty)-T^{2}(\infty)\right|=\left|\frac{4 n+1}{16 n+2}-\frac{-16 n^{2}+4 n+1}{-64 n^{2}+24 n+4}\right|=\left|\frac{1}{4\left(16 n^{2}-6 n-1\right)}\right|=\frac{1}{4} \frac{1}{\left|16 n^{2}-6 n-1\right|}
$$

If $M$ is taken as $\frac{1}{\left|16 n^{2}-6 n-1\right|}, M$ becomes 1 , with $n=0$ and $M<1$, when $n \neq 0$. As a result $\left|T(\infty)-T^{2}(\infty)\right| \leq \frac{1}{4}$. 


\section{Competing interests}

The authors declare that they have no competing interests.

\section{Authors' contributions}

All authors have contributed to all parts of the article. All authors read and approved the final manuscript.

\section{References}

[1] M. Akbaş, On suborbital graphs for the modular group, Bull. London Math. Soc. 33, (2001), 647-652.

[2] M. Beşenk et al., Circuit lengths of graphs for the Picard group, J. Inequal. Appl.,106, (2013), 8 pp.

[3] M. Beşenk, Connectedness of suborbital graphs for a special subgroup of the modular group, Math. Sci. Appl. E-Notes 4 (2016), no. $1,45-54$.

[4] N.L. Biggs and A.T. White, Permutation groups and combinatorial structures, London Mathematical Society Lecture Note Series 33, CUP, Cambridge, 1979.

[5] J. D. Dixon and B. Mortimer, Permutation Groups, Graduate Texts in Mathematics 163, Springer-Verlag, 1996.

[6] B. Ö. Güler et al., Elliptic elements and circuits in suborbital graphs, Hacet. J. Math. Stat. 40 (2011), no. 2, $203-210$.

[7] B. Ö. Güler et al., Suborbital graphs for the group $\Gamma^{2}$, Hacet. J. Math. Stat. 44(5) (2015) 1033-1044.

[8] G. A. Jones, D. Singerman and K. Wicks, The modular group and generalized Farey graphs, London Mathematical Society Lecture Note Series, 160, CUP, Cambridge, 316-338, 1996.

[9] G. A. Jones, Maps on surfaces and Galois groups, Mathematica Slovaca, Vol. 47 (1997), No. 1, 1-33.

[10] P.M. Neumann, Finite Permutation Groups, Edge-Coloured Graphs and Matrices, Topics in Group Theory and Computation, Ed. M. P. J. Curran, Academic Press, London, New York, San Fransisco, 1977.

[11] M. Newman, The Structure of some subgroups of the modular group, Illinois J. Math. 6 (1962), 480-487.

[12] M. Newman, Free subgroups and normal subgroups of the modular group, Illinois J. Math. 8 (1964), 262-265.

[13] M. Newman, Classification of normal subgroups of the modular group, Transactions of the American Math. Society Vol.126 2 (1967), 267-277.

[14] R. A. Rankin, Modular Forms and Functions, Cambridge University Press, 2008.

[15] C.C. Sims, Graphs and finite permutation groups, Math. Z. 95, (1967), 76-86.

[16] D. Singerman, Universal tessellations, Rev. Mat. Univ. Complut. Madrid 1 (1988) 111-123.

[17] T. Tsuzuku, Finite Groups and Finite Geometries, Cambridge University Press, Cambridge, 1982. 